\title{
Mucosal immunity and novel tuberculosis vaccine strategies: route of immunisation- determined T-cell homing to restricted lung mucosal compartments
}

\author{
Rocky Lai, Sam Afkhami, Siamak Haddadi, Mangalakumari Jeyanathan and \\ Zhou Xing
}

Affiliation: McMaster Immunology Research Centre, M. G. DeGroote Institute for Infectious Disease Research, and Dept of Pathology and Molecular Medicine, McMaster University, Hamilton, ON, Canada.

Correspondence: Zhou Xing, Dept of Pathology and Molecular Medicine, and McMaster Immunology Research Centre, McMaster University, 1280 Main Street West, Room MDCL-4012, Hamilton, ON, L8S 4K1, Canada.

E-mail: xingzamcmaster.ca

ABSTRACT Despite the use of bacille Calmette-Guérin (BCG) for almost a century, pulmonary tuberculosis (TB) continues to be a serious global health concern. Therefore, there has been a pressing need for the development of new booster vaccines to enhance existing BCG-induced immunity. Protection following mucosal intranasal immunisation with AdHu5Ag85A is associated with the localisation of antigen-specific T-cells to the lung airway. However, parenteral intramuscular immunisation is unable to provide protection despite the apparent presence of antigen-specific T-cells in the lung interstitium. Recent advances in intravascular staining have allowed us to reassess the previously established T-cell distribution profile and its relationship with the observed differential protection. Respiratory mucosal immunisation empowers T-cells to home to both the lung interstitium and the airway lumen, whereas intramuscular immunisation-activated T-cells are largely trapped within the pulmonary vasculature, unable to populate the lung interstitium and airway. Given the mounting evidence supporting the safety and enhanced efficacy of respiratory mucosal immunisation over the traditional parenteral immunisation route, a greater effort should be made to clinically develop respiratory mucosal-deliverable TB vaccines.

@ERSpublications

Immunisation route determines TB vaccine efficacy based on whether T-cells can enter restricted lung mucosal sites http://ow.ly/M0shT

\section{Introduction}

Mycobacterium tuberculosis is the causative agent of pulmonary tuberculosis (TB) and remains the leading infectious cause of death [1]. Although control of primary M. tuberculosis infection in the lung is strongly correlated with the induction of T-helper cell 1 cellular responses within the lungs, such responses are delayed for up to 3 weeks post-infection $[2,3]$. This leads to unchecked bacterial infection within the host [4]. The only clinically approved TB vaccine, bacille Calmette-Guérin (BCG), accelerates the emergence of T-cell responses in the lung by only a few days [3,5]. Furthermore, BCG-induced immunity wanes over time, so that by adulthood most people are no longer protected from TB. Therefore, the development of vaccines able to further accelerate and enhance existing BCG-mediated T-cell responses has been recognised as a priority in the field of TB vaccine development [6]. To date, there are over a dozen candidate TB vaccines at various stages of testing in the clinical trial pipeline [6], either designed to replace BCG itself or

Received: March 242015 | Accepted after revision: April 212015

Conflict of interest: None declared.

Provenance: Submitted article, peer reviewed.

Copyright OERS 2015. ERR articles are open access and distributed under the terms of the Creative Commons Attribution Non-Commercial Licence 4.0. 
to boost pre-existing BCG responses. In particular, viral-based booster vaccines are among those that have demonstrated great promise in clinical trials [7-9].

\section{Protection against pulmonary TB is determined by the differential distribution of T-cells in lung anatomic compartments following immunisation}

Since the lung is the site of M. tuberculosis entry, examination of immune responses in the lung is of particular importance. Upon exposure to M. tuberculosis, the bacterium is deposited into the lung airway, and infects local macrophage and dendritic cells. After 8-9 days these cells migrate to the lung draining lymph node, where they prime naïve T-cells and provide the activation signals to generate a population of M. tuberculosis-specific T-cells [10]. These cells then travel through the lymphatic circulation into the venous blood circulation, eventually ending up in the pulmonary vasculature (pulmonary artery). Activated antigen-specific T-cells then migrate through the endothelium into the pulmonary interstitium (parenchyma), eventually entering the lung airways where they become long-lived effector memory cells that are able to activate infected macrophage populations for enhanced control of $M$. tuberculosis infection $[2,3]$.

The current knowledge in the field is that the presence of anti-TB T-cells induced by immunisation in the lung airways before or shortly after $M$. tuberculosis infection is critical for protection $[5,11]$. In this regard, the respiratory mucosal route of immunisation is superior to the parenteral route in protecting against pulmonary TB $[12,13]$, because of its ability to induce anti-TB T-cell immunity in the lung airways in addition to inducing $\mathrm{T}$-cell responses in the lung interstitium. In contrast, parenteral immunisation fails to elicit T-cell responses in the airway lumen although it induces T-cells to populate the lung interstitium [12].

Paradoxically, our previous work has demonstrated that despite the generation of fully functional antigen-specific T-cells in the peripheral lymphoid tissues and the lung interstitium, intramuscular immunisation fails to protect against pulmonary TB [14]. It was initially believed that the inability of intramuscular immunisation to protect against pulmonary TB was linked to the absence of T-cells in the airway, which are present following intranasal immunisation. Indeed, adoptive transfer of CD8 T-cells from the spleen of intramuscularly immunised animals into the airway of naïve SCID (severe combined immunodeficiency) mice confers protection against pulmonary $\mathrm{TB}$, highlighting the critical importance of T-cell localisation within the airway for anti-TB immunity [13]. These data, in conjunction with the initial observation that both intramuscular and intranasal immunisation were able to induce anti-TB immunity in the lung as a whole [12], challenge the stereotypical view that the presence of TB-reactive T-cells in the lung before infection equates to protection against pulmonary TB. In other words, the presence of antigen-specific T-cells in the lung interstitium following intramuscular immunisation does not explain the complete lack of protection in the animals immunised via intramuscular route.

The view that the presence of TB-reactive T-cells in the lung is critical for protection ascends from consideration of the lung as a single tissue compartment. In recent years, discrimination of the lung interstitium from the lung airways, by us and others, has led to better understanding of the anatomic distribution of T-cell responses in the lung and its relevance to anti-TB protection. In addition to these two major lung mucosal compartments, however, there is a dense network of pulmonary capillaries underlying the alveoli that forms a third major compartment in the lung [15]. The lung is one of the most highly vascularised organs and holds $\sim 40 \%$ of the total body blood volume at any given time. Thus, blood-borne leukocyte contamination of the lung tissue may have hindered the accurate interpretation of $\mathrm{T}$-cell responses in the lung as a whole or in the lung interstitium.

Historically, a common approach to get around to this problem in animal models is to perfuse the lung via the pulmonary artery before lung mononuclear cell isolation. Our group and others have employed this approach to "exclude" blood-borne leukocytes $[5,12,13]$. However, an intravascular staining approach was recently used to demonstrate that perfusion was far from effective in removing T-cells from the pulmonary vasculature. In fact, up to $97 \%$ of the CD8 T-cells thought to be located in the lung interstitium were present in the lung vasculature following a respiratory viral infection [16]. This observation questions the previous conclusions, including our own, and necessitates revisiting the anatomic distribution of lung T-cell responses following different routes of immunisation.

The availability of the recently developed technique of intravascular staining helps to discriminate pulmonary intravascular and lung tissue T-cell populations for the first time $[16,17]$, and appreciate their relationship with anti-TB protection. In our previous studies we have reported that, regardless of route of immunisation, a large population of anti-TB T-cells can be isolated from perfused mouse lungs [12, 13]. However, when the tissue compartment is discriminated from the vasculature we have found that it is only following intranasal immunisation that antigen-specific T-cells are located primarily within the lung interstitium and airways. In stark contrast, following intramuscular immunisation the majority of such cells are confined to the pulmonary vasculature (unpublished data) and are not present within the lung 
interstitium (fig. 1). The anatomic distribution of T-cells following parenteral immunisation resembles the T-cell distribution in the lung of naive mice, where 99\% of lymphocytes are confined to pulmonary vasculature. These preliminary findings, therefore, provide an updated understanding of the basic mechanisms regarding the localisation of T-cells following different routes of immunisation. Importantly, these findings challenge the conventional concept that the lung interstitium alongside the peripheral lymphoid tissues is a common or unrestricted site for antigen-experienced T-cells to home to.

Interestingly, profiling a variety of chemokine receptors and integrins for their mRNA expression on antigen-experienced T-cells isolated from the total lung of mice immunised via either the respiratory or parenteral route with a viral-vectored TB vaccine also displayed striking differences. Expression of chemokine receptors CCR1, CCR6 and CCR8, and integrins CD103 $\left(\alpha_{\varepsilon} \beta_{7}\right)$ and VLA-1 $\left(\alpha_{1} \beta_{1}\right)$ were many fold increased on antigen-specific T-cells induced by respiratory mucosal immunisation compared with those induced by intramuscular vaccination (unpublished data) (fig. 1). Furthermore, flow cytometry analysis of proteins for these genes on antigen-specific T-cells residing in the lung interstitium and airway lumen following respiratory mucosal immunisation revealed similar expression patterns for these markers on these two populations of T-cells, further supporting that T-cell entry to the lung interstitium is as restricted as entry to the airway lumen. CD103 and VLA-1 expression has been defined as a residential surface marker for the T-cells in nonlymphoid tissues [18-20]. Currently, homing molecules involved in migration of antigen-experienced T-cells to the lung are not well established [21]. This is partly due to the lack of techniques to discriminate intravascular and tissue residing T-cells in the past. Use of the intravascular staining approach will facilitate redefinition of the homing molecules involved in T-cell recruitment to and retention within the lung interstitium and airway lumen.

\section{Effect of local pro-inflammatory signals on the distribution of T-cells in lung anatomic compartments}

The factors that drive the entry of T-cells into different lung mucosal compartments following respiratory mucosal immunisation remain unclear. However, it is well established that innate immune responses play

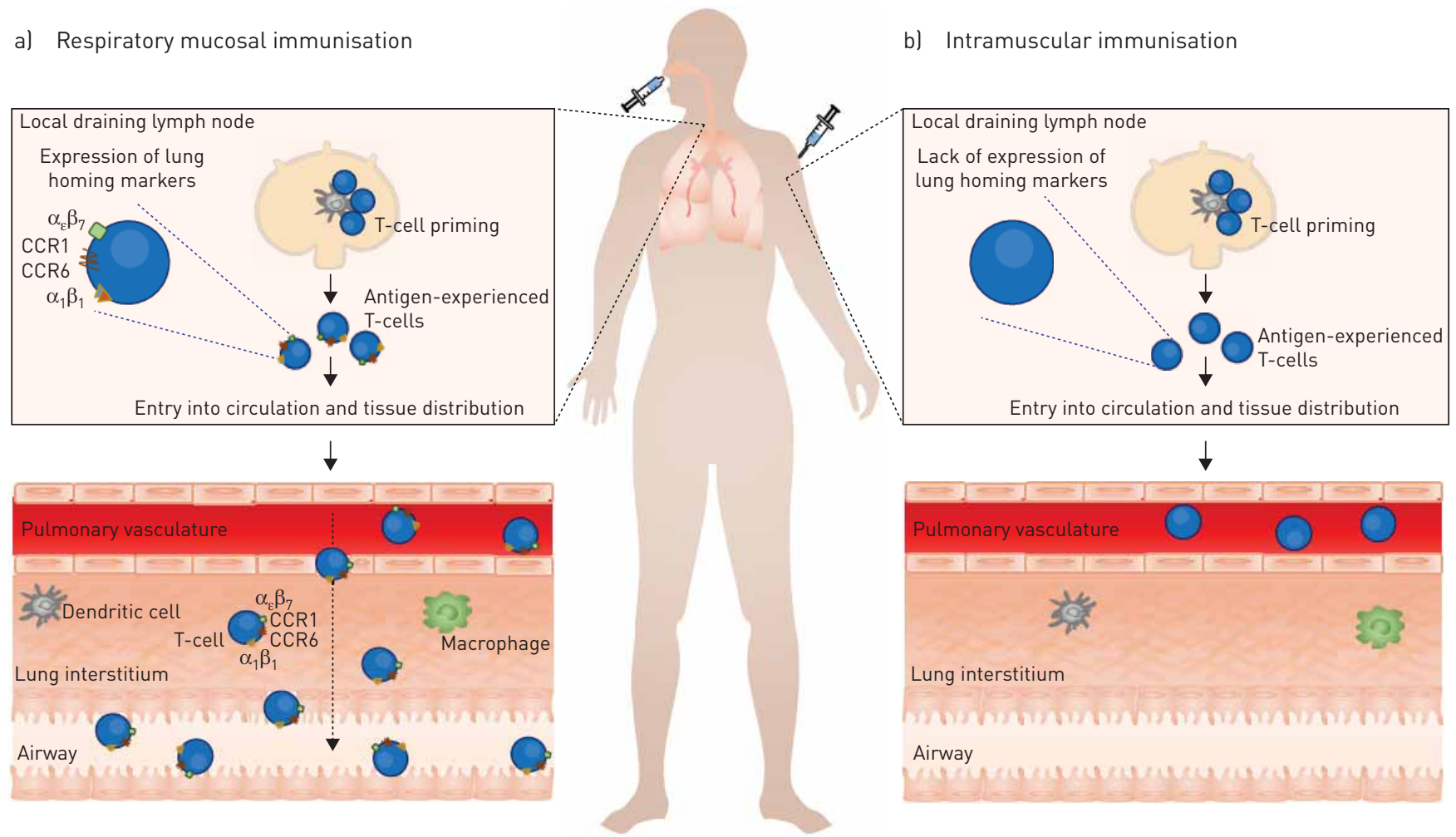

FIGURE 1 Route of immunisation determines whether anti-tuberculosis (TB) T-cells acquire the ability to home to the restricted lung mucosal compartments. Protection against pulmonary TB is associated with the ability of anti-TB T-cells to exit the pulmonary vasculature and gain entry into the restricted lung mucosal compartments. a) T-cells primed within the lung local draining lymph nodes following respiratory mucosal immunisation express various lung homing molecules including $\alpha_{\varepsilon} \beta_{7}$ (CD103), CCR1, CCR6 and $\alpha_{1} \beta_{1}$ (VLA-1). These antigen-experienced T-cells, primed with various lung homing markers, are able to exit the pulmonary vasculature and gain entry into the lung interstitium and airway lumen. Once localised at the site of infection, these cells are able to provide rapid protection against pulmonary TB. b) T-cells primed following parenteral immunisation lack expression of lung homing molecules. Following priming in the local draining lymph nodes, these cells are restricted to the pulmonary vasculature, thus limiting their ability to provide robust anti-TB immunity. 
a large role in the instruction and induction of adaptive T-cell responses. Following immunisation, local dendritic cell populations acquire antigens and subsequently migrate to the local draining lymph node, priming and activating naïve T-cells. It has been suggested that such antigen-presenting cells play an important role in influencing the expression of receptors critical to the migration of T-cells (termed migratory receptors) to specific tissue sites (reviewed by BAATEN et al. [21]).

The anatomical location from which these dendritic cells are derived also seems to influence the trafficking of activated T-cells to specific tissue sites. Extensive evidence demonstrates the ability of local dendritic cell populations to influence the migration of T-cells to the gut and skin [22-25]. Whether this also holds true for T-cell migration to the respiratory tract is less clear. However, a recent study demonstrated that lung dendritic cells were able to preferentially recruit T-cells back to the lung in comparison with dendritic cells derived from other local sites following exposure to inhaled antigens through the induction of CCR4 [26]. However, it is likely that there are other surface molecules involved. In this regard CD8 T-cells primed in the lung draining lymph node were enriched for CCR5 and CXCR3 expression [27]. Furthermore, others have demonstrated that following intranasal vaccination with AdHu5Ag85A, antigen-specific CD8 T-cells localised to the lung expressed high levels of CXCR6 [28]. In addition, we have found CCR1, CCR6, CCR8, CD103 and VLA-1 to be upregulated on activated T-cells by respiratory mucosal, but not by intramuscular, immunisation (unpublished data), suggesting the involvement of multiple molecules in T-cell homing into the lung interstitium and airway. It is tempting to speculate that respiratory mucosal immunisation preferentially activates certain subsets of lung dendritic cells that are able to instruct T-cells to home to the lung interstitium and airway where they are needed.

The superior T-cell responses induced within the lung interstitium and airway lumen by intranasal immunisation may also be attributed to the ability of the viral vaccine backbone to activate the soluble innate immune signals in the local lung microenvironment. Adenoviruses are highly immunogenic, and respiratory mucosal immunisation with AdHu5Ag85A may activate a wide variety of lung local immune responses [29, 30]. In particular, the AdHu5 vector has been shown to elicit potent pro-inflammatory responses including the release of tumour necrosis factor- $\alpha$, interleukin (IL)- 6 and IL-12, as well as the recruitment and activation of macrophages and dendritic cells to the site of infection (unpublished data and $[29,31])$. Indeed, the immunogenic nature of AdHu5 vectors is what makes them an attractive vaccine delivery platform. However, while both intranasal and intramuscular administrations of the vaccine are able to activate local innate responses, only intranasal mucosal immunisation effectively activates local lung innate immune responses. Indeed, we have previously demonstrated that the inoculation of pro-inflammatory agonists, such as Toll-like receptor ligands, into the lung of intramuscularly immunised animals is able to draw antigen-specific T-cells from the peripheral sites into the airway lumen and provide protection against pulmonary TB $[5,14]$. Furthermore, we have recently demonstrated that a robust innate inflammatory response is key for the timely generation of anti-TB T-cell immunity in the lung following primary pulmonary M. tuberculosis infection [10].

\section{Respiratory mucosal vaccination in human clinical trials}

There are ongoing efforts for evaluation of respiratory mucosal vaccination against other respiratory pathogens. In particular, pulmonary delivery of an intranasal live attenuated influenza vaccine has demonstrated high efficacy in children in comparison with the injectable trivalent vaccine [32]. In addition, there have been attempts to explore pulmonary delivery of measles vaccine as an alternative to boost immunity, and this has been demonstrated to be safe and more immunogenic than the injected measles vaccine $[33,34]$.

With regards to $\mathrm{TB}$ vaccines, most current candidates have been developed for and evaluated via the parenteral route of immunisation. MVAAg85A represents one of the most advanced TB vaccine candidates to date. Although the recent phase IIb efficacy trial in South Africa demonstrated that the parenteral route of boosting was ineffective in enhancing protection in BCG-primed infants [9], the demonstrated safety and immunogenicity in a recent phase I MVAAg85A aerosol trial has provided the rationale, and optimism, for respiratory mucosal delivery of a TB vaccine in humans [35].

\section{Conclusion}

Our understanding of what may constitute an effective TB vaccination strategy has increased significantly since BCG was first introduced into human immunisation programmes. The effective boost TB vaccination strategies should aim to generate is memory T-cells that are able to home to the two restricted lung mucosal compartments: the respiratory mucosal surface and the lung interstitium (parenchyma). Such local mucosal tissue-associated T-cells and systemically located T-cells generated by parenteral BCG priming will together provide both local and systemic protection against pulmonary TB. The respiratory mucosal route of immunisation represents the most effective way to generate T-cells capable of homing to the restricted lung mucosal compartments. In comparison, the parenteral route of immunisation activates 
T-cells that are largely trapped only within the pulmonary vasculature and are thus unable to migrate into the site of action, particularly in the early stage of $M$. tuberculosis infection. Increasing numbers of clinical studies to evaluate the respiratory mucosal delivery of TB vaccines are expected in the next few years.

\section{References}

World Health Organization. Global Tuberculosis Report 2014. Geneva, WHO Press, 2014.

2 Shaler CR, Horvath C, Lai R, et al. Understanding delayed T-cell priming, lung recruitment, and airway luminal T-cell responses in host defense against pulmonary tuberculosis. Clin Dev Immunol 2012; 2012: 628293.

3 Cooper AM. Cell-mediated immune responses in tuberculosis. Annu Rev Immunol 2009; 27: 393-422.

4 Davenport MP, Belz GT, Ribeiro RM. The race between infection and immunity: how do pathogens set the pace? Trends Immunol 2009; 30: 61-66.

5 Horvath CN, Shaler CR, Jeyanathan M, et al. Mechanisms of delayed anti-tuberculosis protection in the lung of parenteral BCG-vaccinated hosts: a critical role of airway luminal T cells. Mucosal Immunol 2012; 5: 420-431.

Xing Z, Jeyanathan M, Smaill F. New approaches to TB vaccination. Chest 2014; 146: 804-812.

7 Xing Z, Lichty $\mathrm{BD}$. Use of recombinant virus-vectored tuberculosis vaccines for respiratory mucosal immunization. Tuberculosis (Edinb) 2006; 86: 211-217.

8 Smaill F, Jeyanathan M, Smieja M, et al. A human type 5 adenovirus-based tuberculosis vaccine induces robust $\mathrm{T}$ cell responses in humans despite preexisting anti-adenovirus immunity. Sci Transl Med 2013; 5: 205ra134.

9 Tameris MD, Hatherill M, Landry BS, et al. Safety and efficacy of MVA85A, a new tuberculosis vaccine, in infants previously vaccinated with BCG: a randomised, placebo-controlled phase $2 \mathrm{~b}$ trial. Lancet 2013; 381: 1021-1028.

10 Lai R, Jeyanathan M, Shaler CR, et al. Restoration of innate immune activation accelerates Th1-cell priming and protection following pulmonary mycobacterial infection. Eur J Immunol 2014; 44: 1375-1386.

11 Jeyanathan M, Heriazon A, Xing Z. Airway luminal T cells: a newcomer on the stage of TB vaccination strategies. Trends Immunol 2010; 31: 247-252.

12 Wang J, Thorson L, Stokes RW, et al. Single mucosal, but not parenteral, immunization with recombinant adenoviral-based vaccine provides potent protection from pulmonary tuberculosis. J Immunol 2004; 173: 6357-6365.

13 Santosuosso M, Zhang X, McCormick S, et al. Mechanisms of mucosal and parenteral tuberculosis vaccinations: adenoviral-based mucosal immunization preferentially elicits sustained accumulation of immune protective CD4 and CD8 T cells within the airway lumen. J Immunol 2005; 174: 7986-7994.

14 Santosuosso M, McCormick S, Roediger E, et al. Mucosal luminal manipulation of T cell geography switches on protective efficacy by otherwise ineffective parenteral genetic immunization. J Immunol 2007; 178: 2387-2395.

15 Tschernig T, Pabst R. What is the clinical relevance of different lung compartments? BMC Pulm Med 2009; 9: 39.

16 Anderson KG, Sung $\mathrm{H}$, Skon CN, et al. Cutting edge: intravascular staining redefines lung CD8 $\mathrm{T}$ cell responses. J Immunol 2012; 189: 2702-2706.

17 Sakai S, Kauffman KD, Schenkel JM, et al. Cutting edge: control of Mycobacterium tuberculosis infection by a subset of lung parenchyma-homing CD4 T cells. J Immunol 2014; 192: 2965-2969.

18 Mackay LK, Rahimpour A, Ma JZ, et al. The developmental pathway for $\mathrm{CD}_{103^{+}} \mathrm{CD}^{+}$tissue-resident memory $\mathrm{T}$ cells of skin. Nat Immunol 2013; 14: 1294-1301.

19 Annacker O, Coombes JL, Malmstrom V, et al. Essential role for CD103 in the T cell-mediated regulation of experimental colitis. J Exp Med 2005; 202: 1051-1061.

20 Wakim LM, Gupta N, Mintern JD, et al. Enhanced survival of lung tissue-resident memory $\mathrm{CD} 8^{+} \mathrm{T}$ cells during infection with influenza virus due to selective expression of IFITM3. Nat Immunol 2013; 14: 238-245.

21 Baaten BJ, Cooper AM, Swain SL, et al. Location, location, location: the impact of migratory heterogeneity on T cell function. Front Immunol 2013; 4: 311.

22 Iwata M, Hirakiyama A, Eshima Y, et al. Retinoic acid imprints gut-homing specificity on T cells. Immunity 2004; 21: $527-538$.

23 McCully ML, Ladell K, Hakobyan S, et al. Epidermis instructs skin homing receptor expression in human T cells. Blood 2012; 120: 4591-4598.

24 McCully ML, Moser B. The human cutaneous chemokine system. Front Immunol 2011; 2: 33.

25 Mora JR, Bono MR, Manjunath N, et al. Selective imprinting of gut-homing T cells by Peyer's patch dendritic cells. Nature 2003; 424: 88-93.

26 Mikhak Z, Strassner JP, Luster AD. Lung dendritic cells imprint T cell lung homing and promote lung immunity through the chemokine receptor CCR4. J Exp Med 2013; 210: 1855-1869.

27 Ferguson AR, Engelhard VH. CD8 T cells activated in distinct lymphoid organs differentially express adhesion proteins and coexpress multiple chemokine receptors. J Immunol 2010; 184: 4079-4086.

28 Lee LN, Ronan EO, de Lara C, et al. CXCR6 is a marker for protective antigen-specific cells in the lungs after intranasal immunization against Mycobacterium tuberculosis. Infect Immun 2011; 79: 3328-3337.

29 Tatsis N, Ertl HC. Adenoviruses as vaccine vectors. Mol Ther 2004; 10: 616-629.

30 Muruve DA. The innate immune response to adenovirus vectors. Hum Gene Ther 2004; 15: 1157-1166.

31 Jeyanathan M, Damjanovic D, Shaler CR, et al. Differentially imprinted innate immunity by mucosal boost vaccination determines antituberculosis immune protective outcomes, independent of T-cell immunity. Mucosal Immunol 2013; 6: 612-625.

32 Ambrose CS, Wu X, Knuf M, et al. The efficacy of intranasal live attenuated influenza vaccine in children 2 through 17 years of age: a meta-analysis of 8 randomized controlled studies. Vaccine 2012; 30: 886-892.

33 Dilraj A, Cutts FT, de Castro JF, et al. Response to different measles vaccine strains given by aerosol and subcutaneous routes to schoolchildren: a randomised trial. Lancet 2000; 355: 798-803.

34 Dilraj A, Sukhoo R, Cutts FT, et al. Aerosol and subcutaneous measles vaccine: measles antibody responses 6 years after re-vaccination. Vaccine 2007; 25: 4170-4174.

35 Satti I, Meyer J, Harris SA, et al. Safety and immunogenicity of a candidate tuberculosis vaccine MVA85A delivered by aerosol in BCG-vaccinated healthy adults: a phase 1, double-blind, randomised controlled trial. Lancet Infect Dis 2014; 14: 939-946. 